\title{
Transluminal Contrast Attenuation Gradient Is Associated with Coronary Plaque Vulnerability a Computed Tomography Angiography-based Study
}

\author{
Marius Orzan $^{1}$, Roxana Hodas ${ }^{1}$, Mihaela Dobra ${ }^{1,2}$, Nora Rat ${ }^{1}$, Monica Chitu ${ }^{1,2}$, Imre Benedek ${ }^{1,2}$ \\ ${ }^{1}$ Laboratory of Advanced Research in Multimodal Cardiac Imaging, University of Medicine and Pharmacy, Tîrgu Mureș, Romania \\ ${ }^{2}$ Cardio Med Medical Center, Department of Computational Imaging, Tîrgu Mureș, Romania
}

\section{ABSTRACT}

The aim of this study was to demonstrate that the transluminal contrast attenuation gradient (TAG), a new CT imaging-derived marker of functional significance of a coronary stenosis, is directly associated with the vulnerability degree of atheromatous coronary plaques. Material and methods: This is a prospective study on 21 patients with 30 atheromatous plaques in the coronary arteries, who underwent cardiac computed tomography angiography (CCTA) for assessment of coronary plaques. Results: Twelve plaques were classified as vulnerable (40\%) and 18 plaques (60\%) as non-vulnerable. Plaques associated with a TAG value above 10 HU exhibited in a significantly higher proportion CCTA markers of plaque vulnerability, as compared to plaques in which the attenuation gradient was below $10 \mathrm{HU}$. TAG values $>10$ HU were associated with a higher amount of plaque volume $(107.4 \pm 91.2 \mathrm{~mm} 3$ vs. $56.0 \pm 37.5 \mathrm{~mm} 3, \mathrm{p}$ $=0.009)$, necrotic core $(32.5 \pm 36.9 \mathrm{~mm} 3$ vs. $3.1 \pm 3.2 \mathrm{~mm}, \mathrm{p}=0.0003)$, and fibro-fatty tissue $(17.7 \pm 16.3 \mathrm{~mm} 3$ vs. $4.0 \pm 2.6 \mathrm{~mm} 3, \mathrm{p}=0.0002)$, as compared to those lesions with TAG values below $10 \mathrm{HU}$. Linear regression analysis revealed a significant correlation between TAG values and CCTA features of plaque instability: necrotic core $(r=-0.73, p<0.0001)$, fibrofatty tissue $(r=-0.63, p=0.0002)$, and plaque volume $(r=-0.48, p=0.006)$. Conclusions: In patients with coronary artery disease, contrast attenuation gradient along the coronary plaques, determined by CCTA, correlates with CT markers of plaque vulnerability. Vulnerable coronary plaques are associated with a higher functional significance than the stable ones with a similar anatomic profile.

Keywords: contrast attenuation, computed tomography angiography, coronary artery disease, fractional flow reserve, vulnerable coronary plaque

\section{ARTICLE HISTORY}

Received: July 17, 2017

Accepted: September 10, 2017

\section{CORRESPONDENCE}

Roxana Hodas

Str. Gheorghe Marinescu nr. 38 540136 Tîrgu Mureș, Romania

Tel: +40 265212111

E-mail: roxana.hodas@yahoo.ro

Marius Orzan: Str. Gheorghe Marinescu nr. 38, 540136 Tîrgu Mureș, Romania. Tel: +40 265212 111, E-mail: orzan_marius@yahoo.com Mihaela Dobra: Str. Gheorghe Marinescu nr. 38, 540136 Tîrgu Mureș, Romania. Tel: +40 265212 111, E-mail: d_a_mihaela@yahoo.com Nora Rat: Str. Gheorghe Marinescu nr. 38, 540136 Tîrgu Mureș, Romania. Tel: +40 265212 111, E-mail:ratnora@gmail.com Monica Chitu: Str. Gheorghe Marinescu nr. 38, 540136 Tîrgu Mureș, Romania. Tel: +40 265212 111, E-mail: iuliachitu@yahoo.com Imre Benedek: Str. Gheorghe Marinescu nr. 38, 540136 Tîrgu Mureș, Romania. Tel: +40 265212 111, E-mail: imrebenedek@yahoo.com 


\section{INTRODUCTION}

Despite of recent advances in the field of cardiovascular prevention and rehabilitation, coronary artery disease continues to be associated with high mortality and morbidity rates. ${ }^{1,2}$ Coronary atherosclerosis remains one of the most devastating diseases in the current ages, and its most severe form is represented by acute coronary syndromes (ACS), which are responsible for an increased number of deaths worldwide. ${ }^{1}$ The mortality of patients with acute myocardial infarction can reach $20 \%$ in the absence of appropriate reperfusion treatment. $^{2}$

According to the current definitions, the main forms of ACS are represented by unstable angina, acute myocardial infarction with ST-segment elevation, and acute myocardial infarction without ST-segment elevation. ${ }^{3}$ Despite the differences in the clinical presentation of these three forms, the common substrate that triggers the acute coronary event in all of them is represented by the presence of a vulnerable atheromatous plaque inside the coronary artery. ${ }^{4}$

The concept of plaque vulnerability has been introduced in the literature in the last decades, describing an atheromatous coronary plaque which is exposed to a higher risk of rupture, leading to coronary occlusion and myocardial infarction. ${ }^{5}$ Many studies tried to explain the complex process of plaque vulnerabilization and to characterize the features associated with coronary plaque vulnerability, in an attempt to identify imaging-derived biomarkers that could predict a future coronary event. ${ }^{6,7}$

Imaging studies based on cardiac computed tomography angiography (CCTA) identified several CT features that have been validated as relevant markers of plaque vulnerability. ${ }^{8,9}$ Some of them are related to plaque composition, such as the amount of necrotic core, the plaque burden, the amount of low-density plaque with a CT density below 30 Hounsfield Units (HU), or the presence of spotty calcifications inside the plaque. ${ }^{10}$ Other features are related to morphological characteristics of the diseased vessel, such as the extent of positive remodeling at the site of the atherosclerotic lesion. ${ }^{11}$ However, there are very limited data published so far related to the perturbation of coronary flow at the site of vulnerable plaques and their potential role in plaque vulnerabilization. ${ }^{12}$

When evaluating a coronary artery stenosis, another important feature is represented by the functional significance of the luminal narrowing. ${ }^{13}$ This feature is currently assessed with the help of invasive fractional flow reserve (FFR); however, alternative techniques have been suggested to play a significant role in the assessment of func- tional significance of a stenotic coronary lesion. ${ }^{14,15}$ The attenuation of contrast media along a coronary stenosis at CT examination has been proposed to serve as a reliable measure of the hemodynamic significance of a coronary artery narrowing. ${ }^{16,17}$ However, there are no studies published so far to elucidate the correlation between the attenuation of contrast material along a coronary plaque and the vulnerability of the plaque.

The aim of this study was to demonstrate that a new CT imaging-derived marker of functional significance of a coronary stenosis, represented by the transluminal contrast attenuation gradient (TAG) along the coronary stenosis, is directly associated with the vulnerability degree of atheromatous coronary plaques in patients with coronary artery disease. At the same time, this study aimed to demonstrate the correlation between TAG and other CT imaging-derived vulnerability markers present at the site of the vulnerable coronary lesions.

\section{MATERIAL AND METHODS}

This is a prospective study on 21 patients with coronary artery disease, in whom CCTA was performed for assessment of 30 coronary plaques identified along the entire coronary tree.

The clinical presentation was stable angina, unstable angina, or non-ST-segment elevation acute myocardial infarction, while patients with ST-segment elevation acute myocardial infarction, hemodynamic instability, or renal failure have been excluded from the study. Betablocker administration was used prior to CCTA examination with dose adjustment in order to achieve a sufficiently low heart rate to allow a good quality examination, with the threshold set at $60 / \mathrm{min}$. An intravenous line was used for systemic injection of contrast material at a flow rate of $6.1 \mathrm{~mL} / \mathrm{s}$, followed by a $150 \mathrm{~mL}$ saline flush at the same flow rate.

A Siemens Somatom 128-slice CT equipment (Siemens, Erlangen, Germany) was used for image acquisition and reconstruction. In total, 30 coronary lesions have been identified in the 21 patients and were analyzed using a dedicated software for quantification of plaque components: QANgioCT RE software (Medis, Leiden, The Netherlands), which allowed quantification of different plaque features such as lesion length, plaque volume, mean burden of the atheromatous plaque, the volume of fibro-fatty and fibrotic tissue, necrotic core volume, dense calcium volume, \% stenosis, and mean TAG along the lesion.

TAG, expressed in HU, was defined as the drop in contrast density along the coronary stenosis, calculated as 


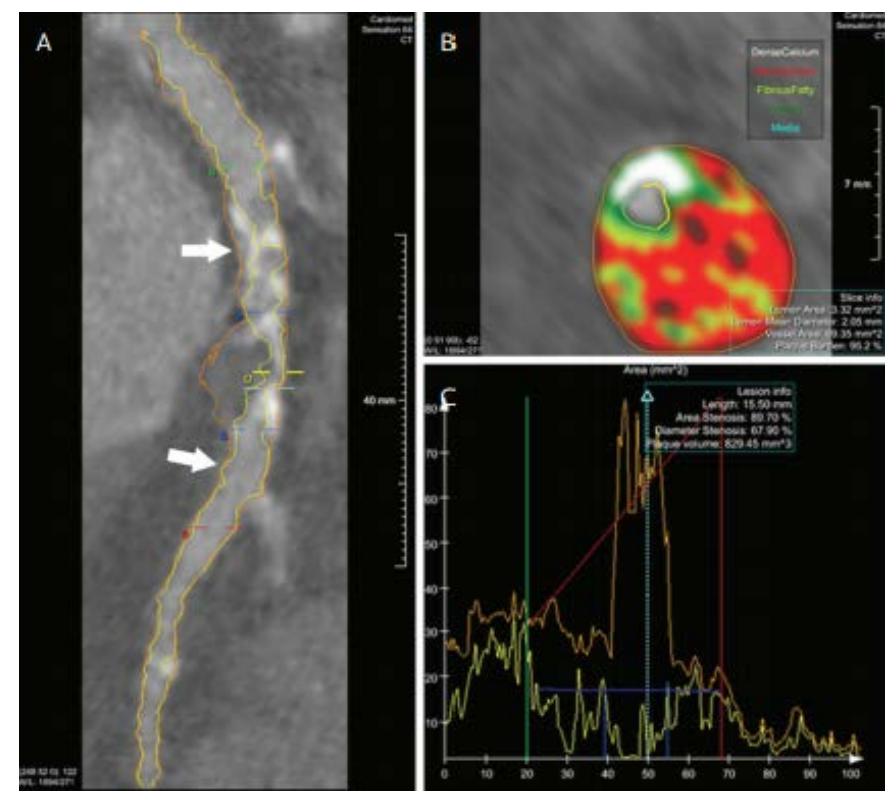

FIGURE 1. Software-based automated computing of a coronary plaque. A - reference markers for determination of transluminal attenuation gradient along the plaque (arrows); B - color coded representation of plaque components; $\mathbf{C}$ - plaque components computing based on contrast density inside the plaque.

the difference in contrast density between the segments located proximally and distally to the stenosis, using as reference points the point located $1 \mathrm{~mm}$ proximally to the plaque and the point located $1 \mathrm{~mm}$ distally to the lesion (Figure 1). The TAG ratio (TAGr) was defined as the ratio between the TAG value across the lesion and the entire length of the lesion and was expressed in $\mathrm{HU} / \mathrm{mm}$.

The study protocol was approved by the ethics committee of the medical center where examinations and analyses were performed, and written informed consent was obtained from each participant included in the study. All the study procedures have been carried out according to the principles stipulated in the Declaration of Helsinki.

TABLE 1. Patient characteristics in the study group

\begin{tabular}{lc}
\hline & $\mathbf{n}(\mathbf{\%})$ \\
\hline Age, years & $60.1 \pm 12.4$ \\
Gender, male & $10(47.6)$ \\
Hypertension & $8(38.1)$ \\
Hyperlipidemia & $12(57.1)$ \\
Obesity (BMI $\left.>25 \mathrm{~kg} / \mathrm{m}^{2}\right)$ & $7(33.3)$ \\
Smoker $*$ & $11(52.3)$ \\
Diabetes & $10(47.6)$ \\
\hline
\end{tabular}

*past or present
GraphPad InStat 3.0 software was used for statistical analysis, using Fisher's exact test (or Student's t-test for age) for comparison of patients and plaque characteristics between the groups. Continuous values were expressed as mean and standard deviation, and the Mann-Whitney test was used for determination of statistical significance in comparison between the groups. Statistical significance was considered for $\mathrm{p}$ values $<0.05$, and all $\mathrm{p}$ values were two-sided.

\section{RESULTS}

Clinical and demographic characteristics of the study population are presented in Table 1 . No statistically significant differences were recorded between the study groups in respect to age, gender, and cardiovascular risk factors (hypertension, obesity, smoking status, dyslipidemia).

From the total of 30 plaques analyzed, 12 were classified as vulnerable $(40 \%)$ and $18(60 \%)$ as non-vulnerable, based on CCTA analysis of plaque features.

CCTA analysis of plaque composition revealed that the following CCTA features were significantly higher in vulnerable plaques as compared to non-vulnerable ones: plaque volume ( $\mathrm{p}=0.1)$, mean plaque burden $(\mathrm{p}=0.009)$, volume of fibro-fatty tissue ( $p=0.009$ ), volume of necrotic core $(p<0.0001)$. On the contrary, dense calcium volume ( $p=0.05)$ was significantly larger in non-vulnerable coronary plaques as compared to vulnerable ones (Table 2).

The severity of coronary artery stenosis, as expressed by the degree of luminal narrowing, was not significantly different between the two groups of plaques $(55.3 \% \pm$ $12.5 \%$ in vulnerable ones and $64.12 \% \pm 14.8 \%$ in non-vulnerable ones, $p=0.1$. Similarly, the length of the lesion was not significantly different $(\mathrm{p}=0.7)$, showing a similar anatomic pattern in the group of vulnerable and nonvulnerable plaques. However, TAG and TAGr values across the vulnerable coronary plaques were significantly higher than those recorded along the non-vulnerable plaques (Figure 2).

At the same time, plaques associated with a TAG value above $10 \mathrm{HU}$ exhibited in a significantly higher proportion CCTA markers of plaque vulnerability, as compared to plaques in which the attenuation gradient was below $10 \mathrm{HU}$. TAG values $>10 \mathrm{HU}$ were associated with a higher amount of plaque volume $(107.4 \pm 91.2 \mathrm{~mm} 3,95 \% \mathrm{CI}$ : $56.9-157.9$ vs. $56.0 \pm 37.5 \mathrm{~mm} 3,95 \%$ CI: $35.2-76.9, \mathrm{p}=$ 0.009), necrotic core ( $32.5 \pm 36.9 \mathrm{~mm} 3,95 \% \mathrm{CI}: 12.1-53.0$ vs. $3.1 \pm 3.2 \mathrm{~mm} 3,95 \% \mathrm{CI}: 1.2-5.0, \mathrm{p}=0.0003)$, and fibrofatty tissue $(17.7 \pm 16.3 \mathrm{~mm} 3$, 95\% CI: $8.6-26.7$ vs. $4.0 \pm$ 
TABLE 2. Plaque characteristics in vulnerable vs. non-vulnerable plaques

\begin{tabular}{lccc}
\hline $\begin{array}{l}\text { Variable } \\
\text { Mean } \pm \text { SD }(95 \% \mathrm{CI})\end{array}$ & $\begin{array}{c}\text { Group 1 } \\
\text { Vulnerable plaques } \\
\mathbf{n}=\mathbf{~ 1 2}\end{array}$ & $\begin{array}{c}\text { Group 2 } \\
\text { Non-vulnerable plaques } \\
\mathbf{n}=\mathbf{1 8}\end{array}$ & p value \\
\hline Lesion length (mm) & $6.3 \pm 4.0(3.7-8.9)$ & $5.9 \pm 2.0(4.9-6.9)$ & 0.7 \\
\% stenosis $(\%)$ & $55.3 \pm 12.5(46.9-63.8)$ & $64.1 \pm 14.8(56.7-71.5)$ & 0.1 \\
Mean plaque burden $(\%)$ & $72.9 \pm 7.2(68.3-77.6)$ & $65.2 \pm 7.4(61.5-68.9)$ & 0.009 \\
Plaque volume $\left(\mathrm{mm}^{3}\right)$ & $113.7 \pm 103.8(47.7-179.7)$ & $60.4 \pm 31.5(44.7-76.1)$ & 0.1 \\
TAG $(\mathrm{HU})$ & $-38.4 \pm 32.4(-59.0-17.8)$ & $6.5 \pm 4.3(8.7-4.4)$ & 0.0004 \\
TAGr $(\mathrm{HU} / \mathrm{mm})$ & $-5.8 \pm 4.3(-8.6-3.1)$ & $-1.2 \pm 0.8(-1.6-0.8)$ & $<0.0001$ \\
Fibrotic tissue volume $\left(\mathrm{mm}^{3}\right)$ & $29.7 \pm 30.7(9.1-50.4)$ & $21.3 \pm 9.6(16.5-26.1)$ & 0.8 \\
Fibrofatty tissue $\left(\mathrm{mm}^{3}\right)$ & $21.6 \pm 17.3(9.9-33.3)$ & $4.6 \pm 3.4(2.9-6.3)$ & 0.009 \\
Calcium volume $\left(\mathrm{mm}^{3}\right)$ & $13.3 \pm 22.0(4.3-30.3)$ & $33.2 \pm 26.0(19.8-46.6)$ & 0.05 \\
Necrotic core tissue $\left(\mathrm{mm}^{3}\right)$ & $43.7 \pm 37.5(18.5-68.9)$ & $2.8 \pm 1.8(1.9-3.8)$ & $<0.0001$ \\
\hline
\end{tabular}

$* \mathrm{TAG}=$ transluminal attenuation gradient

$2.6 \mathrm{~mm} 3,95 \% \mathrm{CI}: 2.5-5.5, \mathrm{p}=0.0002$ ) as compared to the lesions with TAG values below $10 \mathrm{HU}$. However, mean plaque burden was not significantly different in lesions with high TAG values as compared to those with low TAG values $(70.7 \pm 6.8 \mathrm{~mm} 3,95 \%$ CI: $66.9-74.4$ vs. $66.6 \pm 8.9$ mm3, 95\% CI: 61.6-70.9, p = 0.2) (Figure 3).

Linear regression analysis revealed a significant correlation between TAG values and CCTA features of plaque instability: necrotic core $(r=-0.73, p<0.0001)$, fibro-fatty tissue $(r=-0.63, p=0.0002)$, and plaque volume $(r=$ $-0.48, \mathrm{p}=0.006$ ) (Figure 4 ), indicating that plaque vulnerability is associated with a more expressed decrease in contrast density along the lesion as compared with nonvulnerable ones.

\section{DISCUSSIONS}

Assessment of the functional significance of a coronary artery stenosis produced by an atheromatous plaque has become possible in the last years with the help of the recently introduced imaging technologies, such as FFR on invasive or noninvasive route. ${ }^{4}$ Invasive FFR represents today the gold standard for the assessment of hemodynamic significance of a coronary lesion, while noninvasive

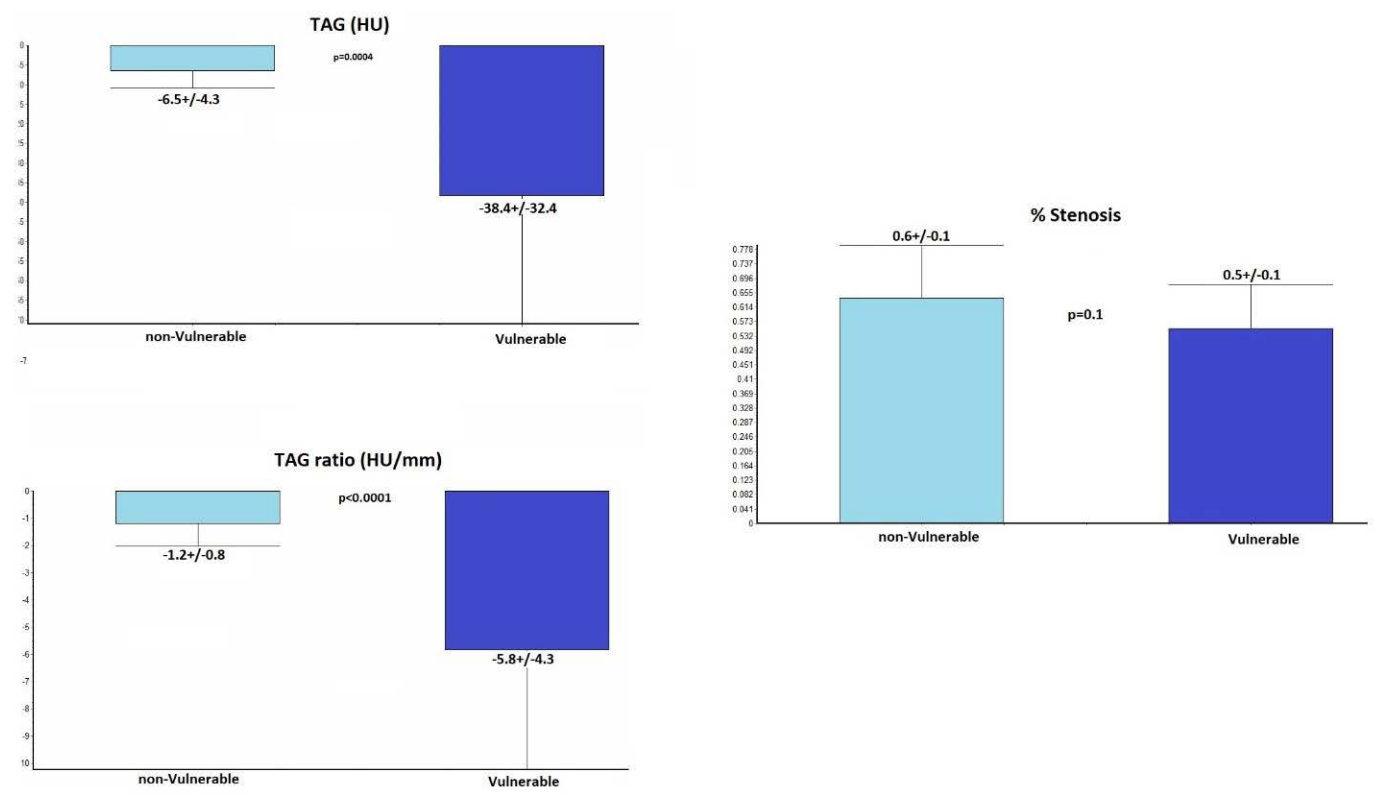

FIGURE 2. Morphologic and functional assessment of vulnerable versus non-vulnerable coronary plaques, as expressed by \% stenosis, TAG, and TAGr 

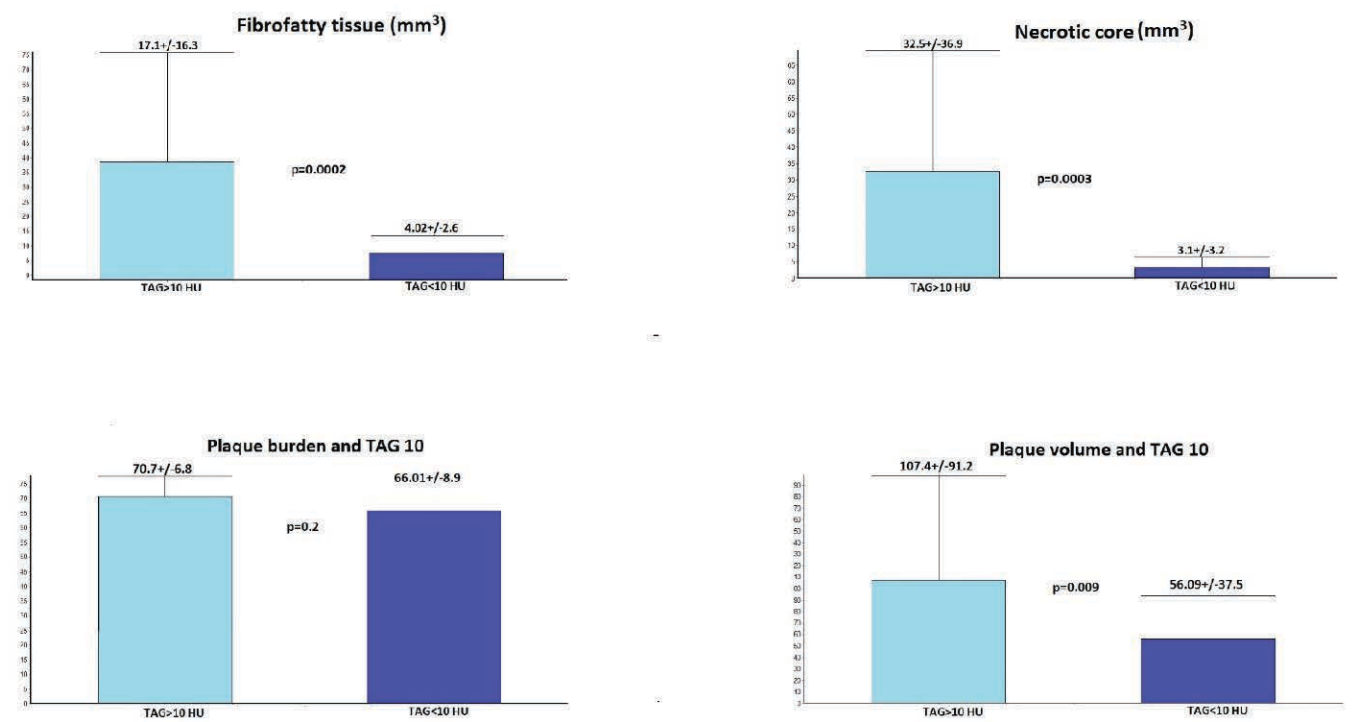

FIGURE 3. CCTA markers of plaque vulnerability in the group of plaques with TAG $>10$ HU versus TAG $<10 \mathrm{HU}$

FFR assessment based on CCTA has been proposed as a promising alternative technique, able to replace the invasive one for this purpose. ${ }^{4}$ At the same time, CCTA is currently validated as an extremely useful tool for the identification of vulnerable coronary plaques based on visualization and quantification of several biomarkers of vulnerability inside the plaque., ${ }^{8,9}$

Determination of TAG by CCTA has been suggested in the last years as a faster and reliable alternative to both invasive and noninvasive FFR for assessing the functional significance of a coronary lesion, based on the concept that the more severe the luminal narrowing is, the higher drop in the contrast density along the coronary plaque will be recorded..$^{18}$

A recently published meta-analysis showed that in patients with coronary artery stenosis, TAG is an independent predictor of major adverse cardiac events (MACE) and can serve as a long-term prognostic tool with a prediction power higher than other clinical predictors or biomarkers. ${ }^{18}$ In recent studies, TAG has shown its superiority compared to invasive FFR in intermediate lesions, showing good sensitivity and specificity for discriminating between significant and non-significant plaques in the group of lesions with stenosis between $30 \%$ and $70 \% .{ }^{15}$
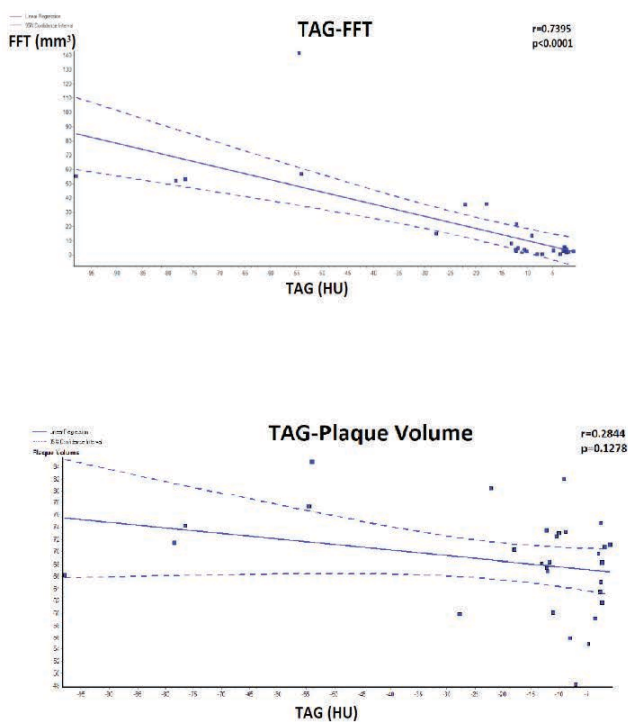
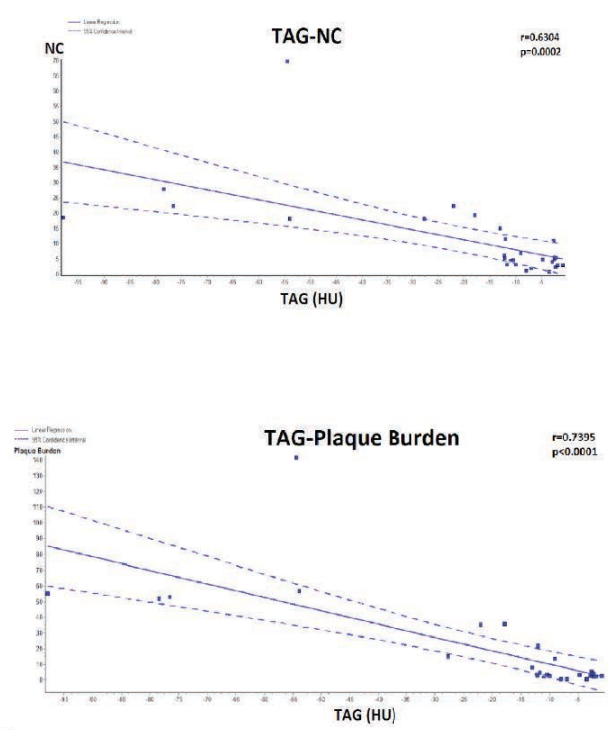

FIGURE 4. Correlation between gradual TAG values and CCTA markers of plaque vulnerability 
However, in the DISCOVER-FLOW and DeFACTO studies, TAG did not show superiority over CT-FFR in the evaluation of stenosis degree. ${ }^{19}$

The role of plaque vulnerability in determination of the functional severity of a stenosis is still unclear, and there are no studies published so far to demonstrate if vulnerable plaques can cause a more severe ischemia as compared with the stable ones. ${ }^{20-23}$

As demonstrated in this study, CCTA can serve as a reliable imaging tool to provide anatomical, quantitative, and functional plaque assessment in a single step. A previous pilot study published by the authors demonstrated a good correlation between CCTA-derived TAG and vulnerability markers in the culprit lesions of patients with acute coronary syndromes.

In this study, we proved that the presence of vulnerability markers at the site of an atheromatous plaque is associated with higher TAG values, reflecting an increased functional significance of vulnerable plaques. ${ }^{12}$ As there were no statistically significant differences in lesion length or stenosis degree between the vulnerable and non-vulnerable lesions, the difference in TAG can be attributed only to the complex mechanisms related to plaque vulnerabilization. The complex local inflammatory mechanisms associated with vulnerable plaques can alter the intracoronary hemodynamic forces, leading to an increased hemodynamic impact on the coronary bed and therefore increased myocardial ischemia, thus explaining the increased MACE rates reported in several studies for lesions with increased TAG. ${ }^{18}$

A large number of studies evaluated the role of CCTA in assessing plaque vulnerability and its associated risk to trigger an acute myocardial infarction. In a study by Benedek et al., the volume of low-density plaque with a CT density below $30 \mathrm{HU}$ was significantly higher in culprit lesions as compared to non-culprit ones. ${ }^{22}$ At the same time, another study demonstrated that this amount of low-density volume is similar to the volume of necrotic core determined by intracoronary imaging using intravascular ultrasound, suggesting that the necrotic core could be quantified by CCTA as the core of the plaque with very low CT density. ${ }^{24}$ However, the functional impact of such a low density core has not been established so far.

According to the authors' knowledge, this is the first study which demonstrated the hypothesis that plaque vulnerabilization can lead to an increased functional significance of the coronary lesion, decreasing coronary perfusion distally to the vulnerable lesion and leading to a more pronounced lesion-specific ischemia.
In a subgroup analysis, we also aimed to assess the vulnerability features in the group of plaques with high TAG versus those with low TAG, using a TAG value of $10 \mathrm{HU}$ as a reference point. We demonstrated that plaques which exhibit attenuation higher than $10 \mathrm{HU}$ along the stenosis present larger volumes of atheromatous plaque, as well as fibro-fatty tissue and necrotic core inside the plaque, markers traditionally associated with vulnerability.

Our study demonstrates that the presence of a large necrotic core is significantly associated with increased TAG values, and this necrotic core volume was significantly higher in plaques with TAG values above $10 \mathrm{HU}$.

In the present study, we succeeded to demonstrate that TAG, a new CCTA imaging-derived parameter characterizing the hemodynamic significance of coronary stenosis, is highly associated with several CCTA markers of plaque vulnerability, such as necrotic core, fibro-fatty tissue, or plaque volume. This study shows that TAG can represent a new biomarker associated with plaque vulnerability, being significantly increased in vulnerable coronary plaques. At the same time, this indicates that in a coronary plaque, the presence of vulnerability features results in an increased functional significance of the stenosis. This probably happens via a complex mechanism which involves exacerbation of local inflammation at the site of the vulnerable plaques that leads to modifications of the local hemorheologic status and alters the coronary flow in the segments located distal to the vulnerable plaque. However, further studies, on larger number of patients, are required to elucidate this hypothesis.

\section{CONCLUSION}

In patients with coronary artery disease, TAG along the coronary plaques, determined by CCTA, correlates with CT-derived markers of plaque vulnerability. Vulnerable coronary artery plaques present a higher functional significance than the stable ones with a similar anatomic profile, indicating that vulnerabilization of a coronary plaque could lead to an increased functional significance of the lesion.

\section{CONFLICT OF INTEREST}

Nothing to declare.

\section{ACKNOWLEDGEMENT}

This research was supported via the research grant no. 103544/2016 - PLaqueIMAGE, contract number 
26/01.09.2016, financed by the Romanian Ministry of European Funds, the Romanian Government and the European Union.

\section{REFERENCES}

1. Dubey G, Verma SK, Bahl VK. Primary percutaneous coronary intervention for acute ST elevation myocardial infarction: Outcomes and determinants of outcomes: A tertiary care center study from North India. Indian Heart J. 2017;69:294298. doi: 10.1016/j.ihj.2016.11.322.

2. Barton GR, Irvine L, Flather $M$, et al. Economic Evaluation of Complete Revascularization for Patients with Multivessel Disease Undergoing Primary Percutaneous Coronary Intervention. Value Health. 2017;20:745-751. doi: 10.1016/j. jval.2017.02.002.

3. Roffi M, Patrono C, Collet JP, et al. ESC Guidelines for the management of acute coronary syndromes in patients presenting without persistent ST-segment elevation. Eur Heart J 2015; 37:267-315. doi: 10.1093/eurheartj/ehv320.

4. Benedek T. The Hemodynamic Impact of Unstable Coronary Plaques - Do We Have the Evidence? Jornal of Cardiovascular Emergencies. 2017;3:59-60. doi: 10.1515/jce-2017-0014.

5. Kwon JE, Lee WS, Mintz GS, et al. Multimodality Intravascular Imaging Assessment of Plaque Erosion versus Plaque Rupture in Patients with Acute Coronary Syndrome. Korean Circ J. 2016;46:499-506. doi: 10.4070/kcj.2016.46.4.499.

6. Tonino PA, Fearon WF, De Bruyne B, et al. Angiographic versus functional severity of coronary artery stenoses in the FAME study fractional flow reserve versus angiography in multivessel evaluation. J Am Coll Cardiol. 2010;55:2816-2821. doi: 10.1016/j.jacc.2009.11.096.

7. De Bruyne B, Pijls NH, Kalesan B, et al. Fractional flow reserveguided PCI versus medical therapy in stable coronary disease. N Engl J Med. 2012;367:1768. doi: 10.1056/NEJMoa1205361.

8. Mollet N, Maffei E, Martini C, et al. Coronary plaque burden in patients with stable and unstable coronary artery disease using multislice CT coronary angiography. Radiol Med. 2011;116:1174-1187. doi: 10.1007/s11547-011-0722-5.

9. Dalager MG, Bøttcher M, Thygesen J, et al. Different Plaque Composition and Progression in Patients with Stable and Unstable Coronary Syndromes Evaluated by Cardiac CT. Biomed Res Int. 2015:401357. doi: 10.1155/2015/401357.

10. Tesche C, DeCecco CN, Caruso D, et al. Coronary CT angiography derived morphological and functional quantitative plaque markers correlated with invasive fractional flow reserve for detecting hemodynamically significant stenosis. J Cardiovasc Comput Tomogr. 2016;10:199-206. doi: 10.1155/2015/401357.

11. De Cecco CN, Caruso D, Baumann S, et al. Coronary CT angiography derived morphological and functional quantitative plaque markers correlated with invasive fractional flow reserve for detecting hemodynamically significant stenosis. J Cardiovasc Comput Tomogr. 2016;10:199-206. doi: 10.1016/j.jcct.2016.03.002.
12. Orzan M, Dobra M, Chitu M. A Comparative Preliminary Study on CT Contrast Attenuation Gradient Versus Invasive FFR in Patients with Unstable Angina. Journal of cardiovascular emergencies. 2017;3:72-78. doi: 10.1515/jce-2017-0012.

13. Dong L, Mintz GS, Witzenbichler B, et al. Comparison of plaque characteristics in narrowings with ST-elevation myocardial infarction (STEMI), non-STEMI/unstable angina pectoris and stable coronary artery disease (from the ADAPT-DES IVUS Substudy). Am J Cardiol. 2015;115:860-866. doi: 10.1016/j. amjcard.2015.01.008.

14. Andreini D, Pontone G, Mushtaq S, et al. A long-term prognostic value of coronary CT angiography in suspected coronary artery disease. JACC Cardiovasc Imaging. 2012;5:690701. doi: 10.1016/j.jcmg.2012.03.009.

15. Cademartiri F, Seitun S, Clemente A, et al. Myocardial blood flow quantification for evaluation of coronary artery disease by computed tomography. Cardiovase Diagn Ther. 2017;7:129150. doi: 10.21037/cdt.2017.03.22.

16. Peng $\mathrm{K}, \mathrm{Xu} \mathrm{N}$, Zhang $\mathrm{L}$, et al. Transluminal attenuation gradient and corrected models in coronary CT angiography for determining stenosis severity: a primary study using dual-source CT. Clin Radiol. 2017;72:508-516. doi: 10.1016/j. crad.2017.01.003.

17. Kim HJ, Kim SM, Choi JH, et al. Influence of scan technique on intracoronary transluminal attenuation gradient in coronary CT angiography using 128-slice dual source CT: multi-beat versus one-beat scan. Int J Cardiovasc Imaging. 2017;33:937946. doi: 10.1007/s10554-017-1078-2.

18. Benz DC, Mikulicic F, Gräni C, et al. Long-term outcome prediction by functional parameters derived from coronary computed tomography angiography. Int J Cardiol. 2017; 243:533-537. doi: 10.1016/j.ijcard.2017.05.083.

19. Boden WE, O'Rourke RA, Teo KK, et al. Optimal medical therapy with or without PCI for stable coronary disease. N Engl J Med. 2007;356:1503-1516. doi: 10.1056/NEJMoa070829.

20. Choi JH, Min JK, Labounty TM, et al. Intracoronary transluminal attenuation gradient in coronary CT angiography for determining coronary artery stenosis. JACC Cardiovasc Imaging. 2011;4:1149-1157. doi: 10.1016/j.jcmg.2011.09.006.

21. Benedek I, Bucur O, Benedek T. Intracoronary infusion of mononuclear bone marrow-derived stem cells is associated with a lower plaque burden after four years. J Atheroscler Thromb. 2014;21:217-229.

22. Benedek T, Gyöngyösi $M$, Benedek I, et al. Multislice computed tomographic coronary angiography for quantitative assessment of culprit lesions in acute coronary syndromes. Can J Cardiol. 2013;29:364-371. doi: 10.1016/j.cjca.2012.11.004.

23. Chow BJ, Kass M, Gagné O, et al. Can differences in corrected coronary opacification measured with computed tomography predict resting coronary artery flow? J Am Coll Cardiol. 2011;57:1280-1288. doi: 10.1016/j.jacc.2010.09.072.

24. Benedek T, Jako B, Benedek I. Plaque quantification by coronary CT and intravascular ultrasound identifies a low CT density core as a marker of plaque instability in acute coronary syndromes. Int Heart J. 2014;55:22-28. 